\title{
The first-generation Daphnia magna linkage map
}

\author{
Jarkko Routtu ${ }^{1 *+}$, Bastiaan Jansen ${ }^{2+}$, Isabelle Colson ${ }^{1,3}$, Luc De Meester ${ }^{2}$, Dieter Ebert ${ }^{1}$
}

\begin{abstract}
Background: Daphnia magna is a well-established model species in ecotoxicology, ecology and evolution. Several new genomics tools are presently under development for this species; among them, a linkage map is a first requirement for estimating the genetic background of phenotypic traits in quantitative trait loci (QTL) studies and is also very useful in assembling the genome. It also enables comparative studies between D. magna and D. pulex, for which a linkage map already exists.

Results: Here we describe the first genetic linkage map of D. magna. We generated 214 F2 (intercross) clonal lines as the foundation of the linkage analysis. The linkage map itself is based on 109 microsatellite markers, which produced ten major linkage groups ranging in size from $31.1 \mathrm{cM}$ to $288.5 \mathrm{cM}$. The total size of this linkage map extends to 1211.6 Kosambi cM, and the average interval for the markers within linkage groups is $15.1 \mathrm{cM}$. The F2 clones can be used to map QTLs for traits that differ between the parental clones. We successfully mapped the location of two loci with infertility alleles, one inherited from the paternal clone (linb1) and the other from the maternal clone (Xinb3).

Conclusions: The D. magna linkage map presented here provides extensive coverage of the genome and a given density of markers that enable us to detect QTLS of moderate to strong effects. It is similar in size to the linkage map of D. pulex.
\end{abstract}

\section{Background}

Daphnia magna has been used in biological research since the 18th century [1]. At the beginning of 2010, a search for Daphnia in ISI Web of Knowledge ${ }^{\mathrm{sm}}$ returned over 12,600 results starting from year 1900 [2]. D. magna is a target of many studies in evolution, ecology and ecotoxicology [3-5]. As a species, it is a relatively large (up to $5 \mathrm{~mm}$ ), widespread and easy to maintain. Normally D. magna reproduces asexually, but it is possible to trigger sexual reproduction under controlled conditions, which is less easy in other members of the Daphnia genus. D. magna is also an ecologically important species in freshwater environments as it is a key grazer of algae while also being the preferred prey of fish [6]. It is widespread in the northern hemisphere, especially in the Palearctic [7]. Moreover, the taxonomy of $D$. magna is relatively straightforward compared to that of the D. pulex and D. longispina

\footnotetext{
* Correspondence: jarkko.routtu@unibas.ch

+ Contributed equally

'Zoologisches Institut, Evolutionsbiologie, Universität Basel, Vesalgasse 1, 4051 Basel, Switzerland

Full list of author information is available at the end of the article
}

species complexes [8]. An advantage of D. magna over $D$. pulex is the relative ease of triggering sexual reproduction and hatching dormant eggs. We are rapidly acquiring new genomics tools for D. magna such as the genome project which all can be found in Daphnia Genomics Consortium webpage [9]. These attributes make this species a very desirable model organism for genetic studies.

The $D$. pulex linkage map is the first and most important reference point in comparing the genetic architecture of $D$. magna. These two species resemble each other phenotypically and share similar broad ecological niches. The divergence between $D$. pulex and D. magna had been estimated from mtDNA sequences to be about 200 MYA [10]. However, recent estimates of divergence time between these species, measured using nuclear genes, is reduced to 7.6 - 15.6 MYA depending on parameters [11].

D. magna is part of the sub-genus Ctenodaphnia, which have been reported to have 10 chromosomes $(2 \mathrm{n}=20)$ [12]. D. pulex (subgenus Daphnia s.s.) has 12 chromosomes, and its genetic map revealed 12 linkage groups $(2 \mathrm{n}=24)$ [13]. Chromosomes in Daphnia are 
small and contracted; thus, karyotypes are difficult to determine [12]. D. magna has no sex-chromosomes because sex determination in Daphnia is environmentally induced, so that the same genotype can be either male or female.

Dominant markers like AFLPs, RFLPs and RAPDs are fast and provide evidence of the overall genetic architecture, but do not effectively disentangle the exact genes responsible for trait differences [14]. Variable numbers of tandem repeats (VNTR) or microsatellite markers, on the other hand, are codominant markers and therefore provide more information. Here we report on a D. magna linkage study using microsatellite markers. The map is part of a larger QTL study aimed at detecting QTLs for various phenotypic traits. The map will also be instrumental in assembling the ongoing D. magna genome project.

The first objective of this study was to generate a linkage map of D. magna based on 109 VNTR marker loci. The second objective was to provide a test case of the D. magna linkage map's potential in QTL mapping. Combined with the published $D$. pulex linkage map, the linkage maps form a first-generation tool for comparative genomics of Daphnia.

\section{Results}

Linkage groups

The sum of Kosambi corrected map units was 1211.6 centiMorgans (cM) for the D. magna map (Table 1). The average interval for the markers in the linkage groups was $15.1 \mathrm{cM}$. The linkage analysis of the F2 panel resulted in 10 linkage groups of more than $30 \mathrm{cM}$ with considerable variation in length (Figure 1). The largest linkage group was $288.5 \mathrm{cM}$, and the tenth largest linkage group was $31.1 \mathrm{cM}$. There were 12 unlinked markers and 7 small linkage groups (5 duplets and 2 triplets) ranging from 0-15.9 cM, indicating that our linkage map is not yet complete. The total length of unlinked small linkage groups was $51.2 \mathrm{cM}$, which is about $4 \%$ of the total map length. From all markers that were used, 74.3 percent showed linkage in the ten largest linkage groups. Many of the unlinked markers as well as the short duplets and triplets showed weak associations to the longer linkage groups (Figure 2). However, they could not be connected to the linkage groups with the critical recombination fraction value of 50 Haldane $c M$ [15] and a LOD score of 3 . The obtained Haldane distances were transformed to Kosambi [16] distances, which provide a better estimate of the real distances between markers by including recombination interference [17]. The EST VNTR markers are also directly mapped genes that are listed in Additional file 1 with their putative functions.
Table 1 Summary of the linkage groups (LG)

\begin{tabular}{|c|c|c|c|c|}
\hline LG & $\begin{array}{c}\text { Number of } \\
\text { markers }\end{array}$ & $\begin{array}{l}\text { Length } \\
\text { Kosambi cM }\end{array}$ & $\begin{array}{l}\text { Average } \\
\text { spacing }\end{array}$ & $\begin{array}{c}\text { Maximum } \\
\text { spacing }\end{array}$ \\
\hline 1 & 17 & 288.5 & 18 & 29.2 \\
\hline 2 & 12 & 181.3 & 16.5 & 36.4 \\
\hline 3 & 11 & 177.6 & 17.8 & 30.8 \\
\hline 4 & 9 & 166.7 & 20.8 & 32.0 \\
\hline 5 & 8 & 108.2 & 15.5 & 22.4 \\
\hline 6 & 6 & 69.9 & 14.0 & 27.3 \\
\hline 7 & 6 & 60.1 & 12.0 & 23.9 \\
\hline 8 & 5 & 43.0 & 10.7 & 20.7 \\
\hline 9 & 5 & 34.0 & 8.5 & 27.8 \\
\hline 10 & 2 & 31.1 & 31.1 & 31.1 \\
\hline 11 & 2 & 15.9 & 15.9 & 15.9 \\
\hline 12 & 3 & 14.0 & 7.0 & 13.9 \\
\hline 13 & 2 & 10.6 & 10.6 & 10.6 \\
\hline 14 & 2 & 7.4 & 7.4 & 7.4 \\
\hline 15 & 2 & 3.1 & 3.1 & 3.1 \\
\hline 16 & 3 & 0.2 & 0.1 & 0.2 \\
\hline 17 & 2 & 0 & 0 & 0 \\
\hline 18 & 1 & 0 & NA & NA \\
\hline 19 & 1 & 0 & NA & NA \\
\hline 20 & 1 & 0 & NA & NA \\
\hline 21 & 1 & 0 & NA & NA \\
\hline 22 & 1 & 0 & NA & NA \\
\hline 23 & 1 & 0 & NA & NA \\
\hline 24 & 1 & 0 & NA & NA \\
\hline 25 & 1 & 0 & NA & NA \\
\hline 26 & 1 & 0 & NA & NA \\
\hline 27 & 1 & 0 & NA & NA \\
\hline 28 & 1 & 0 & NA & NA \\
\hline 29 & 1 & 0 & NA & NA \\
\hline overall & 109 & 1211.6 & 15.1 & 36.4 \\
\hline
\end{tabular}

\section{Transmission ratio distortion (TRD)}

The overall number of heterozygote genotypes was 47.6 percent. The average frequency of heterozygotes did not deviate significantly from the expected distribution of 1:2:1 in F2 intercross design $\left(\chi^{2}=0.19, \mathrm{p}=0.67\right)$. However, individual markers had significant deviations of expected allele distribution (Figure 3, Additional file 2).

TRD was detected in $33 \%$ of the markers. Significant TRDs occurred in several clusters. The majority of the TRD was caused by a lack of heterozygotes (Figure 3), which was pronounced especially in linkage groups 1 and 5 (Figure 3). A lack of homozygotes was seen only in 4 markers belonging to linkage groups 6 and 13 (Figure 3).

\section{Infertility alleles}

Iinb1 clone carried an infertility allele causing dwarfism, red coloration, and no egg production. We called this phenotype red dwarf (RD). Xinb3 clone carried an 


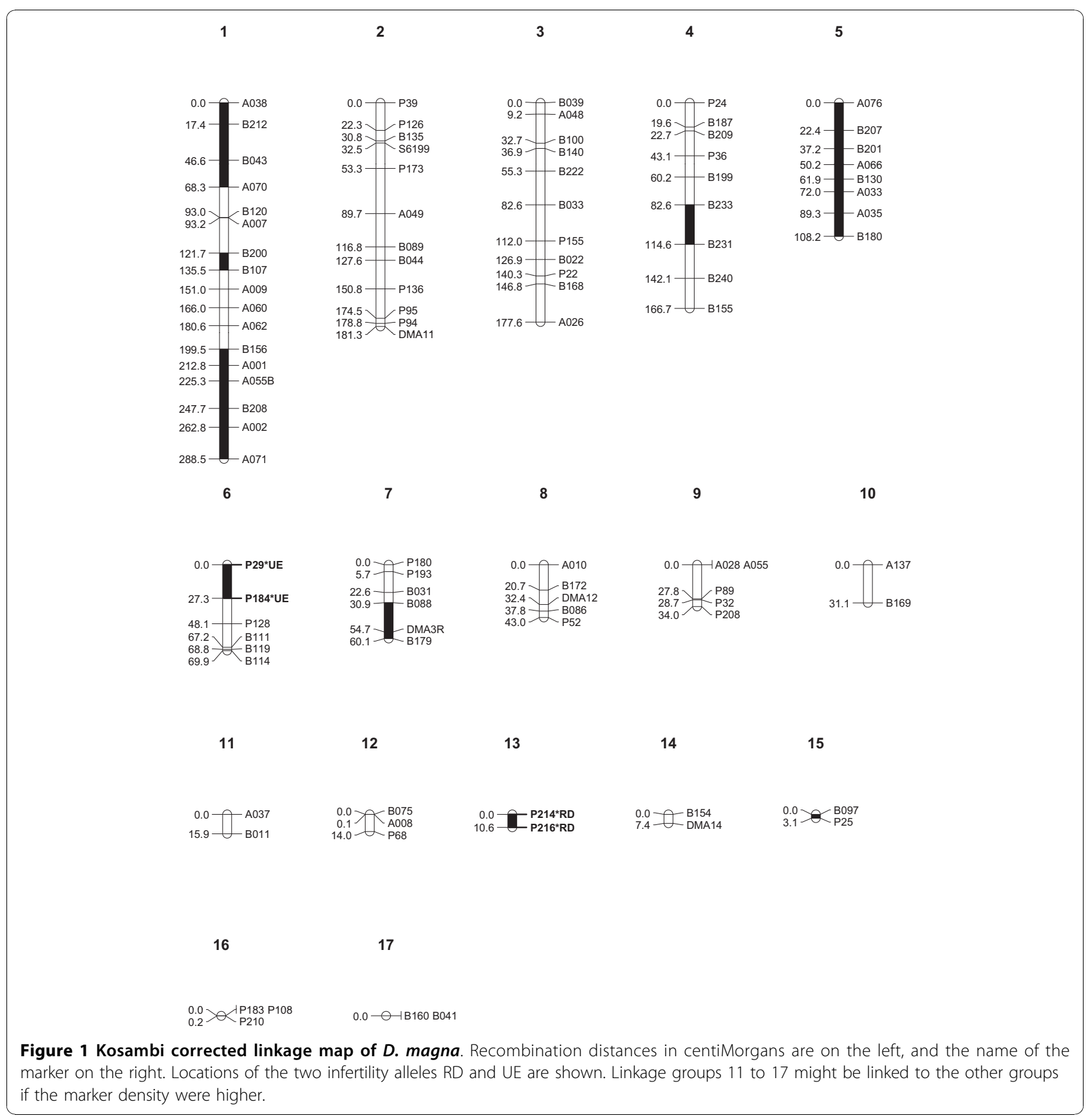

infertility allele causing an otherwise normal phenotype with the exception that asexual eggs would not hatch and no ephippia were produced-here called unviable eggs (UE). RD infertility allele mapped close to marker pair P214 $\left(\mathrm{F}_{1,312}=163.1, \mathrm{p}<0.001\right)$ and P216 $\left(\mathrm{F}_{1,312}=\right.$ 260.8, $\mathrm{p}<0.001$ )(Additional file 2). Both these markers sit on the small linkage group 13 (Table 1, Additional file 2), which is not connected to the main groups. The UE allele mapped close to markers $\mathrm{P} 128\left(\mathrm{~F}_{1,312}=12.3\right.$, $\mathrm{p}=0.001), \mathrm{P} 184\left(\mathrm{~F}_{1,312}=86.9, \mathrm{p}<0.001\right)$ and P29 $\left(\mathrm{F}_{1}\right.$, $312=83.6, \mathrm{p}<0.001)$, which are part of linkage group 6
(Table 1, Additional file 2). The regions in which UE and RD alleles were located were not linked (see Figure 1 ). The infertility allele associations could be traced to the parental genotype/allele. RD infertility allele came from Iinb1 (the father), whereas UE infertility allele came from Xinb3 (the mother). We confirmed the origin of these two infertility alleles by selfing the parental clones, which resulted in approximately $25 \%$ of the infertility phenotypes in the selfed offspring of both parental lines. This is expected for a fully recessive allele being heterozygote in the parental type. The proportions 


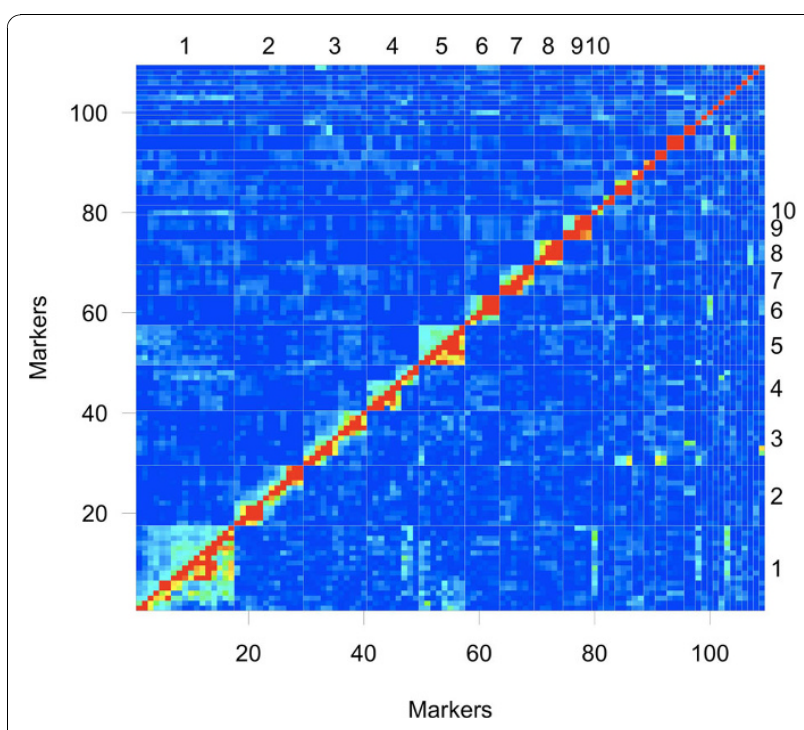

Figure 2 Pair wise recombination fractions and LOD scores Marker (below and left side) and linkage group (above and right side) numbers are at identical positions as in the Figure 1. Above the diagonal; recombination fractions and below the diagonal; corresponding LOD scores. Red indicates high values and blue indicates low values. Green LOD scores are close to significant values.

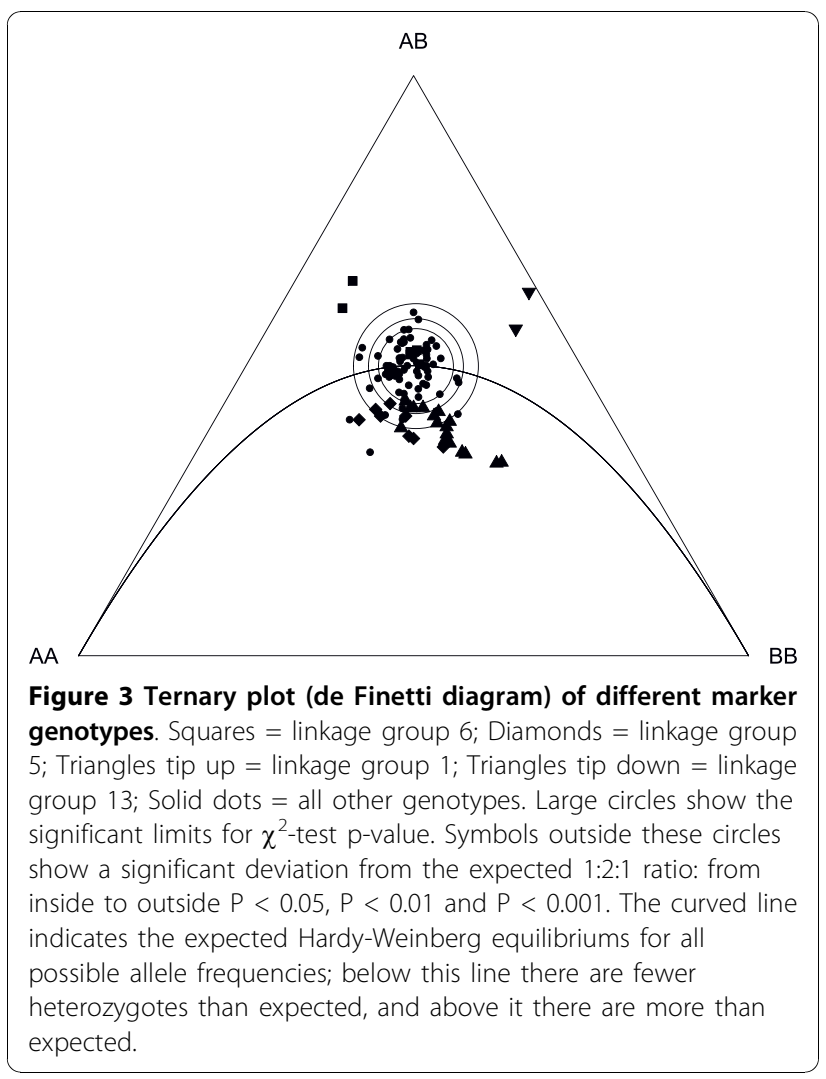

of infertile individuals in F2 individuals were $73 \mathrm{RD}, 78$ UE and 210 fertile. These proportions follow the expected distribution of two recessive alleles in two unlinked loci. When the females showing infertility were excluded from the analysis, the $\chi^{2}$ tests of TRD were P214 $\left(\chi^{2}=42.9, \mathrm{p}<0.001\right)$, P216 $\left(\chi^{2}=61.7, \mathrm{p}<0.001\right)$ for the UE type, and P29 $\left(\chi^{2}=31.0, \mathrm{p}<0.001\right)$ and P184 $\left(\chi^{2}=27.2, p<0.001\right)$ (Additional file 2$)$ for the RD type. But when sterile females (which we could not clone, but still used to assess some of their genotypes) were added to the analysis, the $\chi^{2}$ values were strongly reduced and nonsignificant in the case of the UE type (P214 $\left(\chi^{2}=9.3, \mathrm{p}=0.009\right), \mathrm{P} 216\left(\chi^{2}=12.8, \mathrm{p}=0.002\right)$, P29 $\left.\left(\chi^{2}=2.7, \mathrm{p}=0.25\right), \mathrm{P} 184\left(\chi^{2}=1.9, \mathrm{p}=0.39\right)\right)$. The four markers associated with the two infertility alleles are the four markers that show homozygote deficiency in Fig. 3 (squares and triangles tip down).

\section{Corrected linkage map length and coverage}

The corrected linkage map length was $1519.9 \mathrm{cM}$ for the ten largest linkage groups, which increased to $1692.5 \mathrm{cM}$ when the unlinked smaller linkage groups were added. The coverage of the genome was $86.5 \%$. The expected distance from a closest marker to a random gene was $7.7 \mathrm{cM}$ when all the linkage groups and markers in them were used in the analysis.

\section{Genotyping error}

Genotyping error was estimated to be 1.8 percent, which is slightly higher than the $D$. pulex linkage map estimate. To reduce the impact of potential errors, a small number of marker loci that produced ambiguous results were excluded from the linkage analysis.

\section{Discussion}

The expected number of chromosomes in D. magna is $10(=1 \mathrm{n})$ [12]. Our D. magna linkage map has ten linkage groups exceeding $30 \mathrm{cM}$. These are the best candidates for the ten linkage groups in the D. magna genome. However, the linkage map is a statistical approximation of the recombination pattern and thus not necessarily identical to the physical map.

The linkage map of $D$. pulex consists of 12 linkage groups [13]. The putative ten linkage groups in D. magna differ in some aspects from those of the D. pulex linkage groups. There is one large linkage group of $288.5 \mathrm{cM}$, which is approximately one third larger than the largest linkage group in the $D$. pulex linkage map. The lengths of the two genetic maps are rather close. The D. pulex Kosambi corrected linkage map spanned $1206 \mathrm{cM}$ [13], whereas the D. magna extends 1211.6 Kosambi cM. Even if we exclude the unlinked small linkage groups, the putative linkage map of the ten chromosomes extends $1160.4 \mathrm{cM}$. We used 109 markers 
whereas for the $D$. pulex map 185 markers were used [13]. As a consequence, the average marker interval in the $D$. pulex linkage map is only $7 \mathrm{cM}$, as compared to rather larger $15 \mathrm{cM}$ in the $D$. magna linkage map. However, in the $D$. pulex linkage map, a relatively large number (32\%) of loci are at identical positions, possibly because the number of clonal lines (observed recombination events) used for the $D$. pulex linkage map is lower (129) compared to 214 for D. magna map. In the $D$. magna map, hardly any markers are at identical positions (Fig. 1).

A number of loci are not linked to the putative ten linkage groups of D. magna. This may be a result of incomplete linkage groups, which in turn suggests that the linkage groups extend further than in the present linkage map. There is also some indication of linkage between the unlinked loci themselves. The linkage map of $D$. pulex also resulted in some unlinked loci, but much less than in our study, which is likely to be explained by the smaller average marker distance [13].

We found several cases of TRD, i.e. deviations of the expected genotype distributions in the F2 cross (1:2:1). In two cases, TRD was caused by infertility alleles, which are heterozygote in the parental clones, with both alleles present in the F1 hybrid clone. When the genotypes of the sterile individuals are included into the $\chi^{2}$ test of linkage distortion, the $\chi^{2}$ values are strongly reduced in linkage groups 6 and 13. However, the main cause of TRD in the D. magna linkage map is the lack of heterozygotes (Figure 3). Cristescu et al [13] found that $21 \%$ of their markers showed TRD, resulting in four regions that span about 30 to $70 \mathrm{cM}$. In contrast to the $D$. magna map, TRD in the D. pulex map was mainly caused by a lack of homozygotes [13]. D. magna has ten regions of TRD from $3.1 \mathrm{cM}$ to $89 \mathrm{cM}$, covering $31 \%$ of the markers. An important factor influencing TRD in linkage studies is the genetic distance between the parental lines. Linkage studies on more distantly related parental strains report larger TRD proportions $[18,19]$, with the strongest TRD in QTL studies between species [20]. This may be a consequence of losing synergistic epistatic interactions and resolved genetic conflicts between loci [21]. The parental lines of our panel came from $D$. magna populations that are strongly differentiated ecologically, about 1500 kilometers apart. It is likely that TRD caused by lack of heterozygotes in our study is influenced primarily by the large diversity of the parents, which makes their genomes partially incompatible.

\section{Conclusions}

Based on the overall coverage of the D. magna genome by the linkage map described here and on the similarities of the D. magna and D. pulex linkage maps, we can infer that our D. magna linkage map captures the main elements of the genetic architecture of the D. magna genome. We used infertility alleles present in the parental lines to test that the F2 panel could successfully map the genetic basis of two recessive traits with simple genetics. This linkage map provides a starting point for targeted QTL studies and will be helpful in assembling the D. magna genome. It will need further refining using newly developed markers, and should in time help provide a true physical map of the $D$. magna genome.

\section{Methods}

\section{The F2 intercross design}

The parental clones of our F2 panel were Iinb1 (from Munich, Germany) and Xinb3 (Tvärminne, Finland). Xinb3 is the clone used for the ongoing D. magna genome project. Iinb1 was selfed for one generation, and Xinb3 was selfed for three generations to generate as much inbreeding as possible within the available time. D. magna clones from the Finnish coast self easily, whereas clones from Central Europe did not go easily through the sexual cycle. Male Iinb1 and female Xinb3 were used to produce a F1 hybrid clone. To maximize the range of traits that could be analyzed in the F2 panel, the parental clones were chosen based on divergent phenotypes on parasite resistance and behavioral traits, reflecting the very different ecology of the habitats they were isolated from. The resulting 214 viable F2 clones from the selfed $\mathrm{F} 1$ clone formed the basis for the linkage analysis.

\section{The markers}

DNA from all clones was isolated with peqGOLD Tissue DNA Mini Kit (Peqlab, Erlangen, Germany). Each clone was genotyped for 109 markers: 29 of these were variable number of tandem repeats (VNTRs) developed from expressed sequence tags (ESTs) [22], and 80 were microsatellites (Jansen B, Geldof S, De Meester L Orsini, $\mathrm{L}$ : Isolation and characterization of microsatellite markers in the waterflea Daphnia magna, submitted). The 29 VNTR markers were multiplexed after PCR amplification to groups of non-overlapping marker alleles and analyzed in AB3130xl Sequencer (Applied Biosystems, Foster City, USA). PCR conditions were the same for all loci. An initial denaturation step of 4 minutes at $94^{\circ} \mathrm{C}$ was followed by 35 cycles of $94^{\circ} \mathrm{C}$ for 30 seconds, $52^{\circ} \mathrm{C}$ for 30 seconds, and $72^{\circ} \mathrm{C}$ for 30 seconds, followed by a final extension step of $72^{\circ} \mathrm{C}$ for 4 minutes. The 80 microsatellites were divided in 10 multiplexes. PCR's were done using Qiagen multiplex pcr kit with standard conditions and cycle times (Qiagen, Hilden, Germany). The initial activation step of one minute at $95^{\circ} \mathrm{C}$ was followed with 30 cycles of $94^{\circ} \mathrm{C}$ for 30 seconds, $56^{\circ} \mathrm{C}$ for 30 seconds, and $72^{\circ} \mathrm{C} 30$ seconds followed by a final 
extension step of $60^{\circ} \mathrm{C}$ for 30 minutes. Putative functions were predicted for the EST VNTR markers (Additional file 1).

\section{Linkage analysis}

The initial linkage analysis was done with the program MAPMAKER [23] using commands like; "group", "compare", and "map" for linkage groups of nine or less. For groups larger than nine markers, the command "try" was used to determine the exact position of a marker. The best order was examined with the command "lod table," where genetic distances and LOD scores are displayed as a matrix. Limiting thresholds in the map construction were minimum probability LOD 3 and maximum distance 50 Haldane centiMorgans. Once the markers were assigned to consensus linkage groups, the details of these groups were examined in R/qtl [24], which is an $\mathrm{R}[25,26]$ package for QTL analysis. Linkage analysis was not yet implemented in R/qtl, which was why the initial linkage analysis was done in MAPMAKER. However, we used the est.map() function in R/ qtl to fine-tune the exact positions of the markers, introduce Kosambi correction and detect possible genotyping errors or reversed allele ordering.

\section{Mapping infertility alleles}

Two sterility alleles inherited in the F2 generation as recessive alleles in two nonlinked loci were mapped using the single marker regression option in Windows QTL Cartographer version 2.5 [27]. We used single marker regression, which estimates the position and influence of infertility alleles' on the linkage analysis. We also determined from which parent the infertility alleles originated by examining the segregation pattern in the F2 genotypes and then selfing the parental clones. Fifty animals that hatched from resting eggs in the F2 panel construction of both infertility types were used in the linkage analysis, along with the 214 fertile F2 panel clones.

\section{Ternary plot (de Finetti diagram)}

Ternary plot was used to visually infer the causes of significant TRD. We used R package HardyWeinberg to construct the plot [28]. The $\chi^{2}$-test significance levels $0.05,0.01$ and 0.001 were simulated in HWTernaryPlot () function and drawn separately on the graph.

\section{Corrected linkage map length and coverage}

To estimate the corrected linkage map length and coverage, we followed the method of [21] and [29]. For each linkage group, two cM were added and then multiplied by $(m+1) /(m-1)$, where $m$ is the number of markers in each linkage group. Linkage map coverage $c$ was estimated as $c=1-e^{-2 d n / L}$, with $d$ being the average distance of markers in the linkage map, $n$ the number of markers, and $L$ the length of the linkage map. Finally, we estimated the expected distance of a gene from the nearest random marker locus $E(m)=L /(2 *(n+1))$ $[17,30]$, where $m$ is map units (cM) from the nearest marker, $L$ is the linkage map length, and $n$ is the number of markers.

\section{Genotyping error}

Genotyping error was estimated from a random set of twice genotyped markers, 11 loci and 50 clones genotyped by two different people. The first set was genotyped with a ABI 310 Sequencer (Applied Biosystems, Foster City, USA), and the second set was genotyped with a AB3130xl Sequencer (Applied Biosystems, Foster City, USA).

\section{Additional material}

Additional file 1: EST VNTR markers predicted functions. Name of the marker, EST id in wFleaBase, homolog in protein databases', E-value, predicted biological process and molecular function (excel file).

Additional file 2: Details of the marker loci. Name, linkage group (LG), distance based on recombination fractions in Kosambi centiMorgans $(\mathrm{cM})$, chi square test of linkage distortion $\left(\chi^{2}\right)$, corresponding $p$ value, accession number, forward primer, and reverse primer. The asteriks *** indicate markers that have significant associations with infertility phenotypes (excel file).

\section{Acknowledgements}

Nicolas Boileau, Jürgen Hottinger, Urs Stiefel and Sarah Geldof are acknowledged for help in the laboratory. This study was financially supported by National Institute of Health (USA) Grant 5R24GM078274 and the Swiss National Science Foundation and project G.0269.04 of the National Fund for Scientific Research, Flanders (FWO) and K.U.Leuven Research Fund projects OT/04/23 and GOA/2008/06. Luisa Orsini helped with microsatellite primers. Christoph Haag gave valuable comments.

\section{Author details}

'Zoologisches Institut, Evolutionsbiologie, Universität Basel, Vesalgasse 1, 4051 Basel, Switzerland. 'Katholieke Universiteit Leuven, Charles de Bériotstraat 32, 3000 Leuven, Belgium. 'Bangor University, Bangor LL57 2DG, UK.

\section{Authors' contributions}

JR genotyped EST markers, analysed the data and wrote the manuscript. BJ developed and genotyped the microsatellite markers and contributed to data analysis. IC generated the F2 mapping population, developed and optimized EST markers, and initialized the infertility trait analysis. DE and LDM conceived the study, designed the F2 panel and edited the manuscript. The F2 panel was established in the Ebert lab. All authors read and approved the manuscript.

Received: 6 May 2010 Accepted: 22 September 2010 Published: 22 September 2010

\section{References}

1. Schaffer JC: Die grünen Armpolypen, die geschwänzten und ungeschwänzten zackigen Wasserflöhe und eine besondere Art kleiner Wasseraale Germany: Regensburg1755.

2. ISI Web of Knowledge ${ }^{\mathrm{sm}}$. [http://apps.isiknowledge.com/].

3. Poynton HC, Varshavsky JR, Chang B, Cavigiolio G, Chan S, Holman PS, Loguinov AV, Bauer DJ, Komachi K, Theil EC, Perkins EJ, Hughes O, 
Vulpe CD: Daphnia magna ecotoxicogenomics provides mechanistic insights into metal toxicity. Environmental Science \& Technology 2007, 41:1044-1050

4. Tollrian R, Harvell CD: The Ecology and Evolution of Inducible Defenses Princeton: Princeton University Press 1999.

5. Lampert W, Sommer U: Limnoecology Oxford: Oxford University Press 2007

6. Lampert W: Daphnia: Model herbivore, predator and prey. Polish journal of ecology 2006, 54:607-620.

7. Benzie JAH: Cladocera: The Genus Daphnia (including Daphniopsis). In Guides to the Identification of the Microinvertebrates of the Continental Waters of the World. Edited by: Dumont HJF. Leiden: Backhuys Publisher; 2005:21.

8. Flössner D: Die Haplopoda und Cladocera Mitteleuropas Leiden: Backhuys publishers 2000

9. Daphnia Genomics Consortium webpage. [http://daphnia.cgbindiana.edu/]

10. Colbourne JK, Hebert PDN: The systematics of North American Daphnia (Crustacean: Anomopoda): a molecular phylogenetic approach. Philosophical Transactions of the Royal Society B: Biological Sciences 1996, 351:349-360.

11. Haag CR, MCTaggart SJ, Didier A, Little TJ, Charlesworth D: Nucleotide Polymorphism and Within-Gene Recombination in Daphnia magna and D. pulex, Two Cyclical Parthenogens. Genetics 2009, 182:313-323.

12. Beaton MJ, Hebert PDN: Variation in chromosome numbers of Daphnia (Crustacea, Cladocera). Hereditas 1994, 120:275-279.

13. Cristescu MEA, Colbourne JK, Radivojac J, Lynch M: A microsatellite-based genetic linkage map of the waterflea, Daphnia pulex: On the prospect of crustacean genomics. Genomics 2006, 88:415-430.

14. Schlötterer $\mathrm{C}$ : The evolution of molecular markers-just a matter of fashion? Nature Reviews Genetics 2004, 5:63-69.

15. Haldane JBS: The combination of linkage values and the calculation of distance between loci of linked factors. Journal of Genetics 1919, 8:299-309.

16. Kosambi DD: The estimation of map distances from recombination values. Annals of Eugenics 1944, 12:172-175.

17. Lynch M, Walsh JB: Genetics and Analysis of Quantitative Traits Sunderland, MA: Sinauer Associates, Inc 1998.

18. Hall MC, Willis JH: Transmission Ratio Distortion in Intraspecific Hybrids of Mimulus guttatus: Implications for Genomic Divergence. Genetics 2005 170:375-386

19. McDaniel SF, Willis JH, Shaw AJ: A Linkage Map Reveals a Complex Basis for Segregation Distortion in an Interpopulation Cross in the Moss Ceratodon purpureus. Genetics 2007, 176:2489-2500.

20. Fishman L, Kelly AJ, Morgan E, Willis JH: A Genetic Map in the Mimulus guttatus Species Complex Reveals Transmission Ratio Distortion due to Heterospecific Interactions. Genetics 2001, 159:1701-1716.

21. Turelli M, Orr HA: Dominance, Epistasis and the Genetics of Postzygotic Isolation. Genetics 2000, 154:1663-1679.

22. Colson I, Du Pasquier L, Ebert D: Intragenic tandem repeats in Daphnia magna: structure, function and distribution. BMC Research Notes 2009, 2:206.

23. Lander ES, Green P, Abrahamson J, Barlow A, Daly MJ, Lincoln SE, Newburg L: MAPMAKER: an interactive computer package for constructing primary genetic linkage maps of experimental and natural populations. Genomics 1987, 1:174-181.

24. Broman KW, Wu H, Sen S, Churchill GA: R/qtl: QTL mapping in experimental crosses. Bioinformatics 2003, 19:889-890.

25. Ihaka $R$, Gentleman R: R: A language for data analysis and graphics. Journal of Computational and Graphical Statistics 1996, 5:299-314.

26. R Development Core Team 2008. R: A language and environment for statistical computing [http://www.R-project.org].

27. Wang S, Basten CJ, Zeng Z-B: Windows QTL Cartographer 2.5 Department of Statistics, North Carolina State University, Raleigh, NC 2007 [http://statgen. ncsu.edu/qtlcart/WQTLCart.htm].
28. Graffelman J, Morales J: Graphical tests for Hardy-Weinberg equilibrium based on the ternary plot. Human Heredity 2008, 65:77-84.

29. Chakravarti A, Lasher LK, Reefer JE: A Maximum Likelihood Method for Estimating Genome Length Using Genetic Linkage Data. Genetics 1991, 128:175-182.

30. Martin GB, Williams JGK, Tanksley SD: Rapid identification of markers linked to a Pseudomonas resistance gene in tomato by using random primers and near-isogenic lines. Proceedings of the National Academy of Science 1991, 88:2336-2340.

doi:10.1186/1471-2164-11-508

Cite this article as: Routtu et al:: The first-generation Daphnia magna linkage map. BMC Genomics 2010 11:508.

\section{Submit your next manuscript to BioMed Central and take full advantage of:}

- Convenient online submission

- Thorough peer review

- No space constraints or color figure charges

- Immediate publication on acceptance

- Inclusion in PubMed, CAS, Scopus and Google Scholar

- Research which is freely available for redistribution

Submit your manuscript at www.biomedcentral com/submit
Biomed Central 